Article

\title{
Adaptive Beamformer Combined with Phase Coherence Weighting Applied to Ultrafast Ultrasound
}

\author{
Michiya Mozumi ${ }^{1}$ and Hideyuki Hasegawa ${ }^{2, *}$ \\ 1 Faculty of Engineering, University of Toyama, Toyama 930-8555, Japan; s1470267@ems.u-toyama.ac.jp \\ 2 Graduate School of Science and Engineering, University of Toyama, Toyama 930-8555, Japan \\ * Correspondence: hasegawa@eng.u-toyama.ac.jp; Tel.: +81-76-445-6741
}

Received: 14 December 2017; Accepted: 25 January 2018; Published: 30 January 2018

\begin{abstract}
Ultrafast ultrasound imaging is a promising technique for measurement of fast moving objects. In ultrafast ultrasound imaging, the high temporal resolution is realized at the expense of the lateral spatial resolution and image contrast. The lateral resolution and image contrast are important factors determining the quality of a B-mode image, and methods for improvements of the lateral resolution and contrast have been developed. In the present study, we focused on two signal processing techniques; one is an adaptive beamformer, and the other is the phase coherence factor (PCF). By weighting the output of the modified amplitude and phase estimation (mAPES) beamformer by the phase coherence factor, image quality was expected to be improved. In the present study, we investigated how to implement the PCF into the MAPES beamformer. In one of the two examined strategies, the PCF is estimated using element echo signals before application of the weight vector determined by the adaptive beamformer. In the other strategy, the PCF was evaluated from the element signals subjected to the mAPES beamformer weights. The performance of the proposed method was evaluated by the experiments using an ultrasonic imaging phantom. Using the proposed strategies, the lateral full widths at half maximum (FWHM) were both $0.288 \mathrm{~mm}$, which was better than that of $0.348 \mathrm{~mm}$ obtained by the mAPES beamformer only. Also, the image contrasts realized by the MAPES beamformer with the PCFs estimated before and after application of the mAPES beamformer weights to the element signals were $5.61 \mathrm{~dB}$ and $5.32 \mathrm{~dB}$, respectively, which were better than that of $5.14 \mathrm{~dB}$ obtained by the mAPES beamformer only.
\end{abstract}

Keywords: adaptive beamformer; coherence factor; lateral spatial resolution; image contrast

\section{Introduction}

Ultrafast ultrasound imaging with parallel beamforming [1] is now frequently used for functional imaging such as the measurement of shear wave propagation [2-4], evaluation of myocardial function [5-7], and blood flow imaging [8-14]. However, the parallel beamforming degrades the lateral spatial resolution and image contrast because unfocused transmit beams are used to illuminate a wide region [2]. The lateral resolution and contrast are important factors determining image quality. Therefore, signal processing methods for improvement of the lateral resolution and contrast are demanded for the ultrafast ultrasound imaging. Spatial compounding $[15,16]$ and synthetic aperture imaging $[17,18]$ have been used as such signal processing methods. However, the spatial compounding and the synthetic aperture imaging require multiple transmissions and, thus, the frame rate is degraded. It would be beneficial if the spatial resolution were improved without compromising the temporal resolution.

Recently, adaptive beamforming has been studied and used in many applications $[19,20]$. In the delay-and-sum (DAS) beamforming, element echo signals are delayed based on the geometrical information of the focal point and each transducer element. The weights applied to element echo 
signals in the DAS beamforming are predetermined and independent of the received data. On the other hand, in the adaptive beamformer, weights are adaptively optimized using received echo signals [19]. The minimum variance (MV) beamforming [20], a kind of adaptive beamformer, determines the weights to minimize the power of the beamformer output while maintaining unit gain of a signal of interest. Many researchers have previously attempted to introduce the MV beamformer to the field of the medical ultrasound imaging [21-23], and the MV beamformer provides a significant improvement of the lateral spatial resolution. Also, Synnevåg et al. demonstrated the capability of the MV beamformer to compensate for the degradation of the lateral resolution arising from the parallel beamforming [24]. Blomberg et al. proposed the amplitude and phase estimation (APES) beamformer [25], which eliminates the desired signal from the spatial covariance matrix by DAS beamforming, where the desired signal means the signal which is coherent with the echo from the focal point. We modified the APES beamforming for more accurate estimation of the desired signal by considering the directivity of the array transducer element to discard sub-array averaging [26-29].

On the other hand, adaptive weighting methods based on the coherence factor have also been studied for improvement of the lateral spatial resolution. The coherence factor works as a metric to evaluate the focusing error in receive beamforming, and it is evaluated from echo signals received by individual transducer elements. $\mathrm{Li}$ and $\mathrm{Li}$ proposed the generalized coherence factor (GCF) [30], which is the ratio of the energy of the low spatial frequency components of element echo signals to the total energy. The direct current (DC) component and the high-frequency components were regarded as the coherent and incoherent signals, respectively and, hence, the GCF represents the degree of the focusing error. Camacho et al. proposed the phase coherence factor (PCF) and the sign coherence factor (SCF) [31], which are evaluated from the phases of the delay compensated echo signals in the DAS beamforming. The PCF is determined by the phase variance among received signals, and the SCF is determined by changes in polarities of the received signals.

For further improvement of the lateral resolution and contrast, in the present study, we examined two strategies to combine the adaptive beamforming and the coherence-based imaging. In one of the two examined strategies, the PCF is estimated using element echo signals before application of the weight vector determined by the adaptive beamformer. In the other strategy, the PCF was evaluated from the element signals subjected to the MAPES beamformer weights.

We also tried to solve a problem in the PCF. Some researchers have already tried to combine the coherence factor with the MV beamforming [32-34]. In those studies, the coherence factor is evaluated from echo signals received by individual transducer elements. However, echoes from a diffuse scattering medium will be suppressed when the PCF is estimated from echo signals received by individual transducer elements because there are many echoes with similar amplitudes and they interfere with each other. To overcome such a problem, we previously proposed the phase coherence imaging with sub-aperture beamforming [35-37]. Sub-aperture beamforming reduces the effect of interference among echoes from diffuse scattering medium, and the visibility of the diffuse scattering medium in phase coherence imaging was improved. In the present study, we also tried to implement the PCF into the modified APES beamformer with sub-aperture beamforming. The performance of the proposed method was evaluated by the experiments using an ultrasound imaging phantom.

\section{Materials and Methods}

\subsection{Modified Amplitude and Phase Estimation (mAPES) Beamforming [26]}

The complex ultrasound signals received by individual transducer elements in an ultrasound array probe are defined as follows:

$$
\mathbf{S}=\left(s_{0}, s_{1}, \cdots, s_{M-1}\right)^{\mathrm{T}},
$$


where ${ }^{\mathrm{T}}$ and $M$ denote the transpose and the number of transducer elements, respectively, and $s_{m}(m=0,1, \cdots, M-1)$ is the complex echo signal received by the $m$-th transducer element. The output signal $u$ is expressed as follows:

$$
u=\mathbf{w}^{\mathrm{H}} \mathbf{S}
$$

where ${ }^{\mathrm{H}}$ and $\mathbf{w}$ are Hermitian operator and the weight vector applied to the received echo signals, respectively. Let us define the spatial covariance matrix by $\mathbf{R}=\mathrm{E}\left[\mathbf{S S}^{\mathrm{H}}\right]$, where $\mathrm{E}[\cdot]$ denotes the expectation. In the APES beamforming, the weight $\mathbf{w}_{\mathrm{APES}}$ is expressed as follows:

$$
\mathbf{w}_{\mathrm{APES}}=\frac{\mathbf{Q}^{-1} \mathbf{a}}{\mathbf{a}^{\mathrm{H}} \mathbf{Q}^{-1} \mathbf{a}^{\prime}}
$$

where $\mathbf{Q}=\mathbf{R}-\mathbf{G G}^{\mathrm{H}}, \mathbf{G}=\left[g_{0}, g_{1}, \cdots, g_{M-1}\right]^{\mathrm{T}}$, and $\mathbf{a}$ is the steering vector expressed as follows:

$$
\mathbf{a}=\left(\begin{array}{c}
a_{0} \\
a_{1} \\
\vdots \\
a_{M-1}
\end{array}\right)=\left(\begin{array}{c}
\exp \left(-j \frac{2 \pi f_{0} \sqrt{\left(x_{0}-x_{f}\right)^{2}-z_{f}^{2}}}{c_{0}}\right) \\
\exp \left(-j \frac{2 \pi f_{0} \sqrt{\left(x_{1}-x_{f}\right)^{2}-z_{f}^{2}}}{c_{0}}\right) \\
\vdots \\
\exp \left(-j \frac{2 \pi f_{0} \sqrt{\left(x_{M-1}-x_{f}\right)^{2}-z_{f}^{2}}}{c_{0}}\right)
\end{array}\right)
$$

where $f_{0}$ and $c_{0}$ are the ultrasonic center frequency and speed of sound, respectively. The vector $\mathbf{G}$ corresponds to the desired signal from the receiving focal point $\left(x_{f}, z_{f}\right)$, and $g_{m}(m=0,1, \cdots, M-1)$ is estimated as follows [26]:

$$
g_{m}=\frac{D\left(\theta_{m}\right) \mathbf{a}^{\mathrm{H}} \mathbf{S}}{\sum_{i=0}^{M-1} D\left(\theta_{i}\right)}
$$

where

$$
\begin{aligned}
& D\left(\theta_{m}\right)=\frac{\sin \left(\frac{\pi l}{\lambda} \sin \theta_{m}\right)}{\frac{\pi l}{\lambda} \sin \theta_{m}}, \\
& \theta_{m}=\tan ^{-1}\left(\frac{x_{f}-x_{m}}{z_{f}}\right) .
\end{aligned}
$$

In our previous study, the outputs of the sub-aperture beamformers were used instead of $\mathbf{S}$ in Equation (2) [26]. The output $y_{k}$ of the $k$-th sub-aperture $(k=0,1, \cdots, K-1)$ consisting of $M_{\text {sub }}=M / K$ elements is expressed as follows:

$$
\begin{gathered}
y_{k}=\left(a_{M_{\mathrm{sub}} \cdot k}^{*} a_{M_{\mathrm{sub}} \cdot k+1}^{*}, \cdots, a_{M_{\mathrm{sub}} \cdot k+M_{\mathrm{sub}}-1}^{*}\right)\left(\begin{array}{c}
s_{M_{\mathrm{sub}} \cdot k} \\
s_{M_{\mathrm{sub}} \cdot k+1} \\
\vdots \\
s_{M_{\mathrm{sub}} \cdot k+M_{\mathrm{sub}}-1}
\end{array}\right)=\mathbf{a}_{k}^{\mathrm{H}} \mathbf{s}_{k}, \\
u_{\mathrm{mAPES}}=\mathbf{w}_{\mathrm{mAPES}}^{\mathrm{H}} \mathbf{Y}
\end{gathered}
$$

where

$$
\begin{gathered}
\mathbf{Y}=\left(y_{0}, y_{1}, \cdots, y_{K-1}\right)^{\mathrm{T}}, \\
\mathbf{w}_{\text {mAPES }}=\frac{\mathbf{C}^{-1} \mathbf{J}}{\mathbf{J}^{\mathrm{H}} \mathbf{C}^{-1} \mathbf{J}^{\prime}}
\end{gathered}
$$




$$
\begin{gathered}
\mathbf{C}=\mathbf{Y} \mathbf{Y}^{\mathrm{H}}-\mathbf{V} \mathbf{V}^{\mathrm{H}}, \\
\mathbf{V}=\left(v_{0}, v_{1}, \cdots, v_{K-1}\right)^{\mathrm{T}}, \\
v_{k}=\frac{b_{k} \sum_{i=0}^{K-1} y_{i}}{\sum_{i=0}^{K-1} b_{i}}, \\
b_{k}=\sum_{i=0}^{M_{\mathrm{sub}}-1} D\left(\theta_{M_{\mathrm{sub}} \cdot k+i}\right),
\end{gathered}
$$

where $\mathbf{J}$ is a $K$ dimensional vector of ones.

\subsection{Gaussian Phase Coherence Factor ( $g P C F)$}

The PCF is originally defined by the standard deviation of the phases of echo signals received by individual transducer elements [31] and used for weighting the beamformed echo signals to suppress echoes with focusing errors. In our previous study, the gPCF was proposed to enhance the effect of the PCF [36]. The gPCF is expressed as follows:

$$
\mathrm{gPCF}=\exp \left\{\rho \times\left(\frac{\sigma}{\sigma_{0}}\right)^{2}\right\}
$$

where $\rho$ is the control parameter, which was set at 3 in the present study [36]. Also, $\sigma_{0}$ is the nominal standard deviation of $\pi / 3^{0.5}$ of the uniform distribution between $-\pi$ and $\pi$ [31], and $\sigma$ is the standard deviation among phases of delay-compensated echo signals received by individual transducer elements or outputs from sub-aperture beamformers. When an echo is coming from the receiving focal point and sound speed in medium is homogeneous, no focusing error occurs and the gPCF is estimated to be 1 . On the other hand, when an echo is coming from the out-of-focal point, phase variance increases and the gPCF falls to 0 . In our previous study, the outputs of the sub-aperture beamformers were prepared and, then, the phase variance was estimated using outputs of the sub-apertures to suppress interferences from the out-of-focus echoes [35]. In the present study, the gPCF is estimated using the output $y_{k}$ of each sub-aperture defined in Equation (8) (i.e., output signals before applying the adaptive beamformer weights).

\subsection{Modifiled APES Beamformer Weighted by gPCF}

In the present study, two strategies were examined to implement the gPCF into the mAPES beamformer. In both strategies, the outputs of the mAPES beamforer were weighted by the gPCF, but the gPCFs was estimated differently. The schematic diagrams are shown in Figure 1. In one of the examined strategies, as illustrated in Figure 1a, the gPCF was estimated from the outputs of sub-aperture beamformers before application of the MAPES beamformer weights, i.e., the gPCF is estimated using $y_{k}(k=0,1, \cdots, K-1)$ in Equation (8). In another strategy, the output signals from the sub-aperture beamformer weighted by the MAPES beamfomer weights were used to estimate the $\mathrm{gPCF}$, i.e., the gPCF is estimated using $y_{k} \cdot w_{\mathrm{mAPES}, k}(k=0,1, \cdots, K-1)$, where $w_{\mathrm{mAPES}, k}$ is the $k$-th element of $\mathbf{w}_{\text {mAPES }}$ defined in Equation (11). To make a B-mode image, either Figure $1 \mathrm{a}$ or Figure $1 \mathrm{~b}$ is adopted to estimate the gPCF applied to the output of the adaptive beamformer. Performances of such two procedures were examined in the subsequent section. 

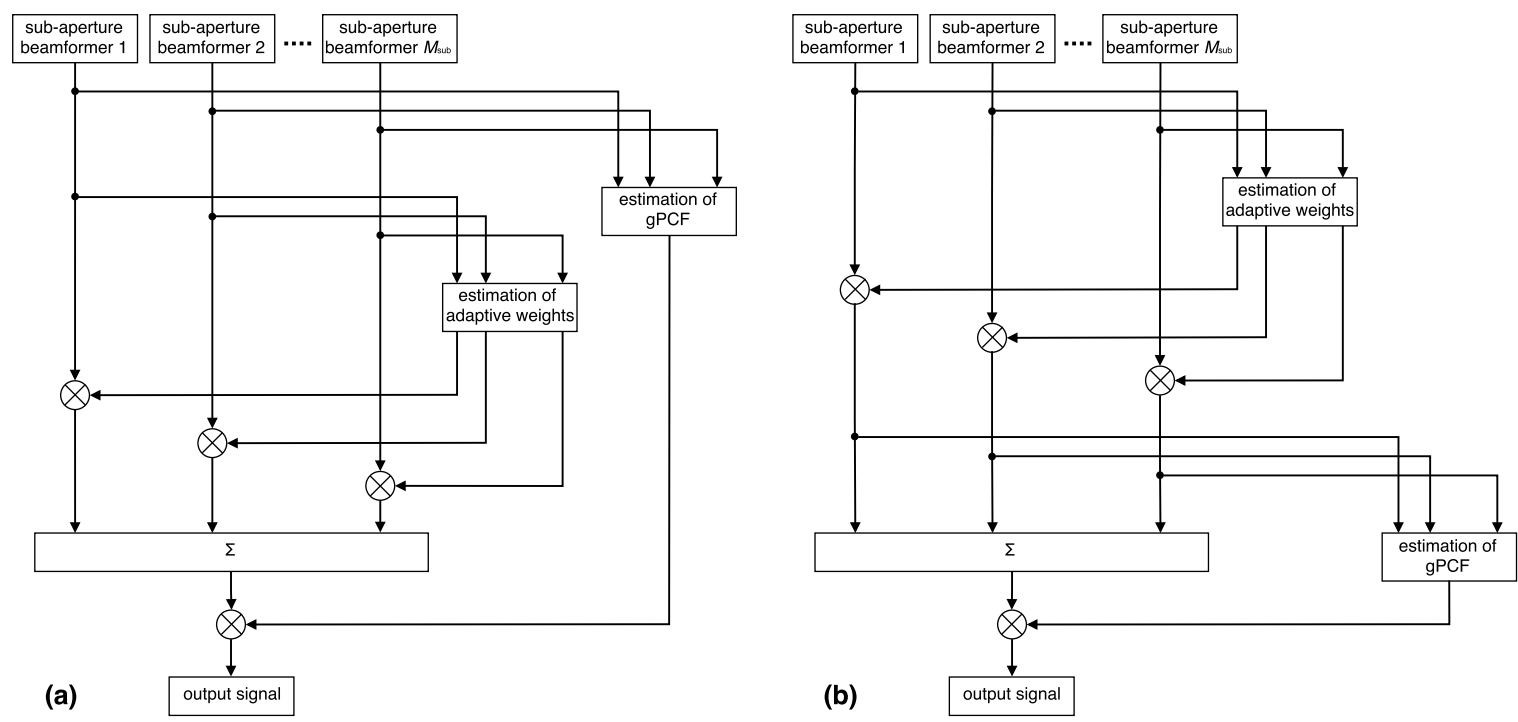

Figure 1. Illustration of weighting procedure using Gaussian Phase Coherence Factor (gPCF) estimated (a) before and (b) after applying adaptive beamformer weights to outputs of sub-aperture beamformers.

\subsection{Experimental Methods and Evaluation Metrics}

\subsubsection{Experimental Setup}

In the present study, an ultrasound imaging phantom (model 040GSE, CIRS, Norfolk, VA, USA) was used for evaluation of image quality. A linear array ultrasonic probe at a nominal center frequency of $7.5 \mathrm{MHz}$ was used. The element pitch of the linear array and the number of the transducer elements were $0.2 \mathrm{~mm}$ and 192, respectively. Ultrasonic echo signals received by individual transducer elements were acquired by a custom-made ultrasound scanner (RSYS0002, Microsonic, Tokyo, Japan) at a sampling frequency of $31.25 \mathrm{MHz}$. The beamforming procedure was performed off-line using the numerical analysis software MATLAB (The MathWorks Inc., Natick, MA, USA). The transmit-receive procedure is described in [9]. In the present study, plane waves were emitted using 96 transducer elements, and then, 24 receiving beams were created in response to one emission. By repeating such a transmit-receive procedure four times, $24 \times 4=96$ receiving beams were created at intervals of $0.2 \mathrm{~mm}$. The frame rate achieved under such transmit-receive response condition was $1302 \mathrm{~Hz}$ at the pulse repetition frequency of $5208 \mathrm{~Hz}$.

\subsubsection{Spatial Resolution}

The spatial resolution was evaluated using the lateral full width at half maxima obtained from the amplitude profile of an echo from a point scatterer (fine string in the phantom) [26].

\subsubsection{Contrast}

Image contrast $C$ was evaluated as follows [26]:

$$
C=\frac{\left|\mu_{b}-\mu_{l}\right|}{\left(\mu_{b}+\mu_{l}\right) / 2}
$$

where $\mu_{b}$ and $\mu_{l}$ are mean gray levels in background and lesion, respectively. In the present study, an anechoic cyst phantom was adopted as the lesion, and a diffuse scattering medium was adopted as the background. 


\subsubsection{Peak-to-Speckle Ratio}

In the present study, the peak-to-speckle ratio is defined as the ratio of a peak gray level at a strong scatterer (fine string in the phantom) to the mean gray level in diffuse scattering medium.

\section{Results}

\subsection{Basic Experimental Results Using Phantom}

Figure $2 \mathrm{a}-\mathrm{d}$ show B-mode images of a string phantom obtained by the conventional DAS beamforming, the mAPES beamforming without gPCF, and those with gPCFs estimated before and after applying the adaptive beamformer weights, respectively.
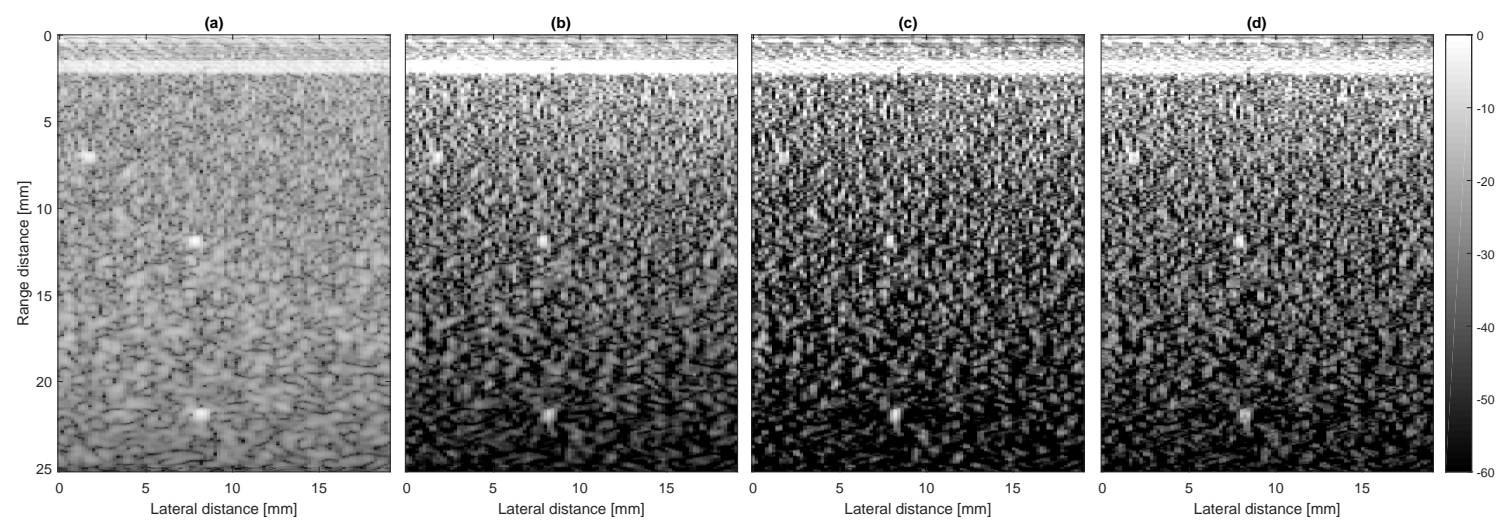

Figure 2. B-mode images of string phantom obtained (a) with delay-and-sum (DAS); (b) with modified amplitude and phase estimation (mAPES) beamforming without gPCF, and mAPES beamforming with gPCFs evaluated (c) before and (d) after applying the adaptive beamformer weights.

In the phantom used for this experiment, three point targets were placed at different axial depths. Figure $3 \mathrm{a}, \mathrm{b}$ show the lateral amplitude profiles with respect to point targets at axial depths of $12 \mathrm{~mm}$ and $22 \mathrm{~mm}$ in Figure 2a-d.
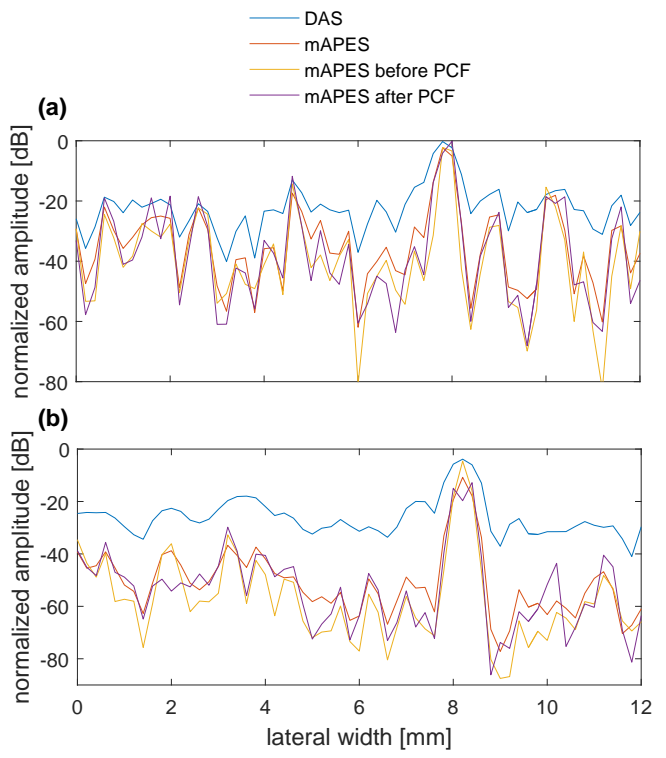

Figure 3. Lateral amplitude profile obtained from point target at axial depths of (a) $12 \mathrm{~mm}$ and (b) $22 \mathrm{~mm}$ in Figure 2. 
In Figure $3 a, b$, the lateral amplitude profile obtained with the proposed method was sharpened and speckles obtained with the proposed method were resolved as compared to those obtained with the conventional mAPES beamformer. The lateral full widths at half maxima of the lateral amplitude profiles shown in Figure 3a obtained by DAS, mAPES without gPCF, and those with gPCFs estimated before and after applying the adaptive beamformer weights were 0.560, 0.392, 0.356, and $0.344 \mathrm{~mm}$, respectively. Also, the lateral full widths at half maxima of the lateral amplitude profiles shown in Figure 3b obtained by DAS, mAPES without gPCF, and those with gPCFs estimated before and after applying the adaptive beamformer weights were $0.668,0.348,0.288$, and $0.288 \mathrm{~mm}$, respectively. Figure $4 \mathrm{a}, \mathrm{b}$ show that the phases of the outputs of the sub-aperture beamformers before and after applying the adaptive beamformer weights, respectively, which was obtained at range and lateral positions of $22 \mathrm{~mm}$ and $3.60 \mathrm{~mm}$, respectively.

(a)

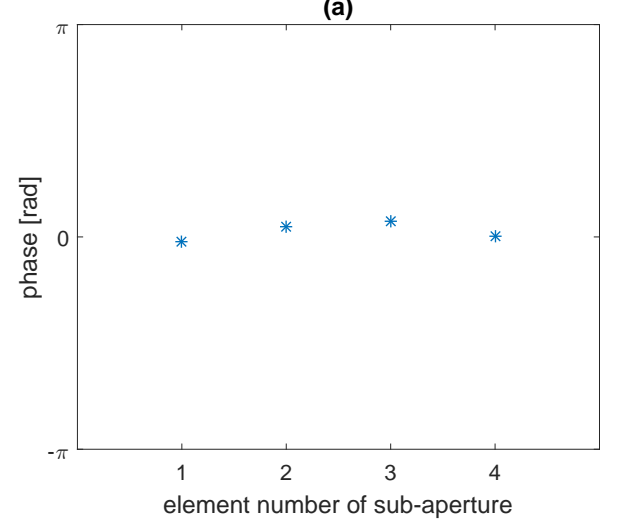

(b)

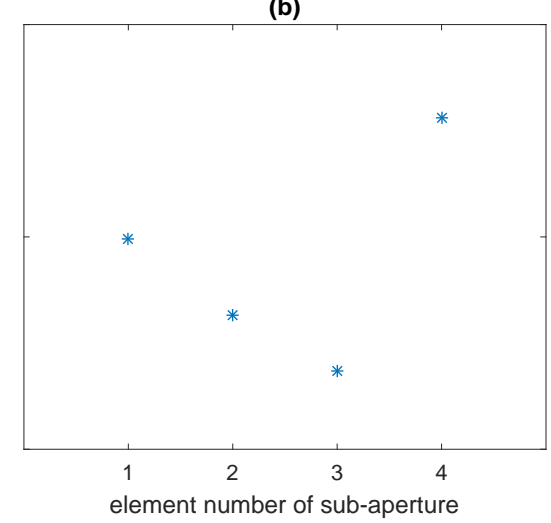

Figure 4. Phase of the signal obtained by each sub-aperture element (a) before and (b) after applying the adaptive weights.

These results show that the phases shown in Figure $4 \mathrm{~b}$ deviate among the sub-apertures, whereas those shown in Figure 4a were well aligned.

Figure $5 \mathrm{a}-\mathrm{d}$ show B-mode images of a cyst phantom obtained by the DAS beamforming, the mAPES beamforming without the gPCF, and those with the gPCFs estimated before and after applying the adaptive beamformer weights, respectively.
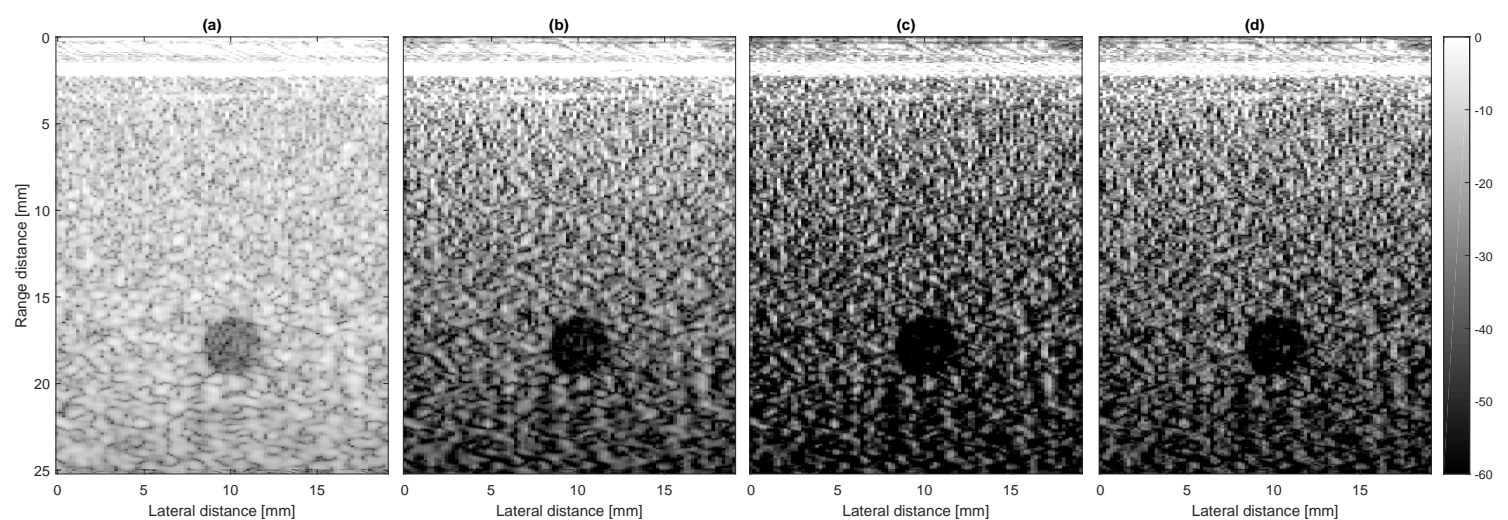

Figure 5. B-mode images of cyst phantom obtained (a) with DAS; (b) with mAPES beamforming without gPCF, and mAPES beamforming with gPCFs evaluated (c) before and (d) after applying the beamformer weights. 
In this experiment, an anechoic cyst was embedded in the phantom. A rectangular region (from 16.8 to $19.2 \mathrm{~mm}$ in the range direction, from 9 to $11 \mathrm{~mm}$ in the lateral direction) was regarded as the lesion, and another rectangular region (from 16.8 to $19.2 \mathrm{~mm}$ in the range direction, from 5 to $7 \mathrm{~mm}$ in the lateral direction) was regarded as background. Contrasts of the B-mode image obtained by DAS, mAPES without gPCF, and those with gPCFs estimated before and after applying the adaptive beamformer weights were 4.05, 5.14, 5.61, and $5.32 \mathrm{~dB}$, respectively. Experimental results show that image contrast was improved by the proposed method.

Figure $6 \mathrm{a}, \mathrm{b}$ show B-mode images of the wire phantom and the cyst phantom obtained by the adaptive beamforming combined with the PCF estimated without the sub-aperture beamforming.
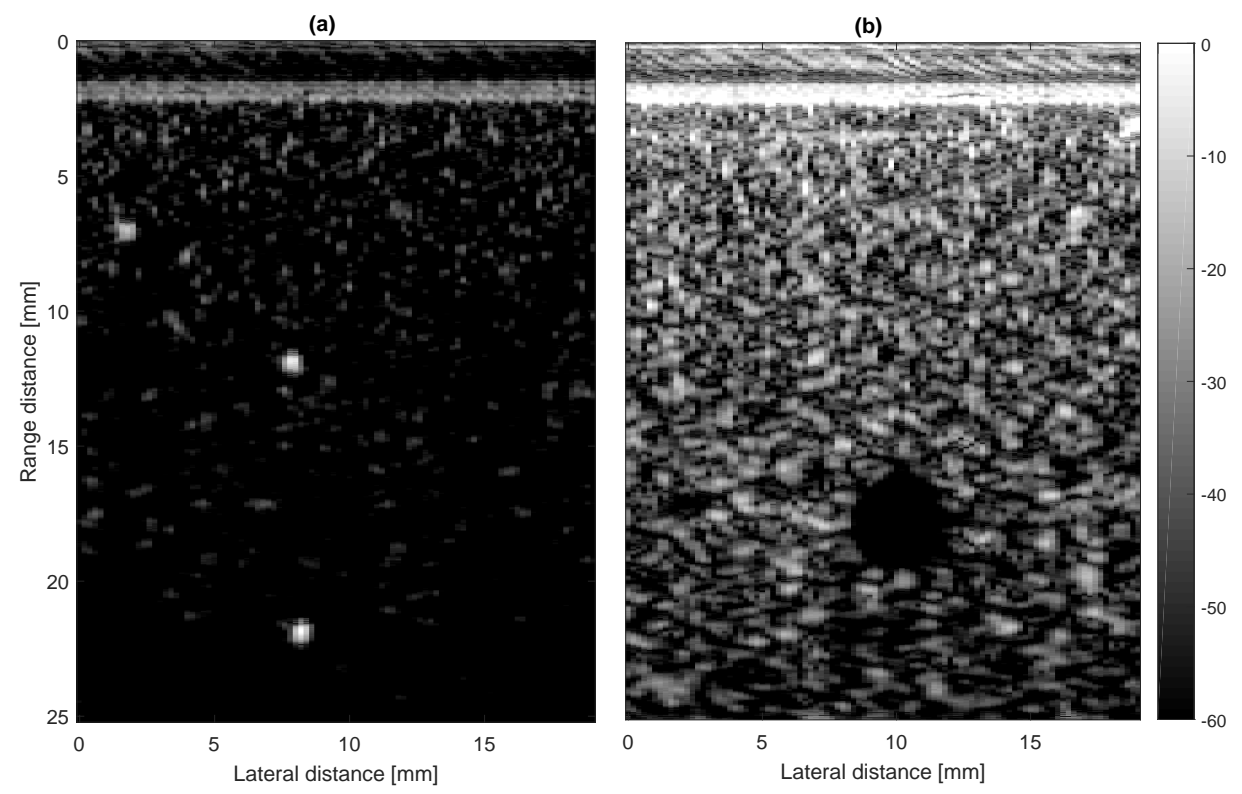

Figure 6. B-mode images of (a) wire phantom and (b) cyst phantom obtained by mAPES beamformer with gPCF estimated in a conventional way, i.e., gPCF was estimated without sub-aperture beamforming. Element echo signals before application of mAPES beamformer weights were used for estimation of gPCF.

In Figure 6a,b, the gPCF was estimated before applying the adaptive beamforming weights. The lateral full widths at half maxima of point targets at depths of $12 \mathrm{~mm}$ and $22 \mathrm{~mm}$ in Figure 6a were $0.356 \mathrm{~mm}$ and $0.280 \mathrm{~mm}$, respectively, and the contrast of the B-mode image in Figure $6 \mathrm{~b}$ was estimated to be $5.99 \mathrm{~dB}$. As shown in Figure 6a, echoes from diffuse scattering medium are suppressed by the PCF when sub-aperture beamforming was not used, and such a negative effect of the PCF estimated from element echo signals can be reduced by sub-aperture beamforming as shown in Figure 3. Figure 7a,b show the lateral resolution and the peak-to-speckle ratio at each number of sub-apertures obtained from point targets at axial depths of $12 \mathrm{~mm}$ and $22 \mathrm{~mm}$, respectively. In the present study, mean gray levels in a rectangular region (from 10.8 to $13.2 \mathrm{~mm}$ in the range direction, from 3 to $5 \mathrm{~mm}$ in the lateral direction) and another rectangular region (from 20.8 to $23.2 \mathrm{~mm}$ in the range direction, from 3 to $5 \mathrm{~mm}$ in the lateral direction) were used for evaluation of the peak-to-speckle ratios in Figure 7a,b, respectively. The gPCF obtained without sub-aperture beamforming corresponds to the number of sub-apertures of 72 .

In Figure $7 a, b$, the lateral full widths at half maxima do not vary with increasing the number of sub-apertures, whereas the peak-to-speckle ratios increase. Experimental results show that the gray level in diffuse scattering medium becomes relatively low under a large number of sub-apertures and, hence, the speckles are not visualized well when the proposed method is used without sub-aperture beamforming. 


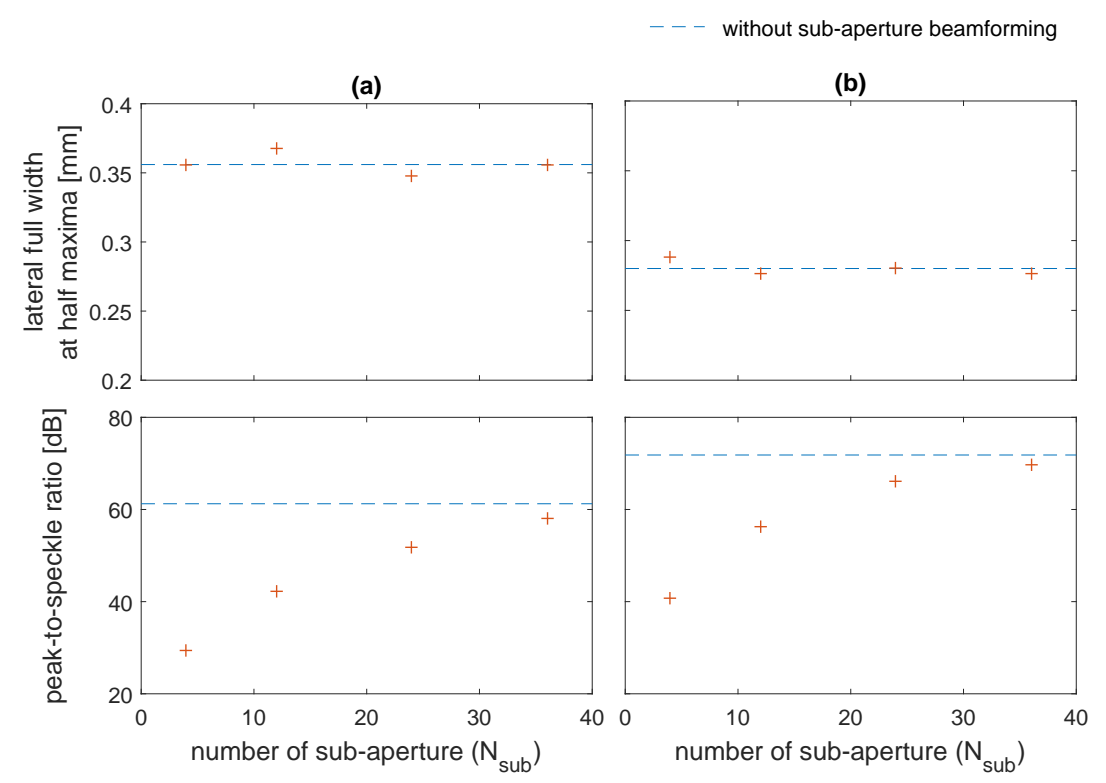

Figure 7. Changes in (top) lateral full widths at half maxima and (bottom) peak-to-speckle ratio obtained by the proposed method with gPCF estimated before applying the adaptive beamformer weights. Results were obtained with respect to point targets at axial depths of (a) $12 \mathrm{~mm}$ and (b) $22 \mathrm{~mm}$.

\subsection{In Vivo Measurement of Human Carotid Artery}

In the present study, the feasibility of the proposed method under in vivo condition was evaluated by measurement of a human carotid artery. Figure 8a-d show B-mode images of the carotid artery obtained by the DAS beamforming, the mAPES beamforming without gPCF, and those with $\mathrm{gPCFs}$ estimated before and after applying the adaptive beamformer weights, respectively.

In Figure $8 c, d$, the wall of the carotid artery was depicted more clearly and sharpened along the lateral direction than Figure 8a,b.
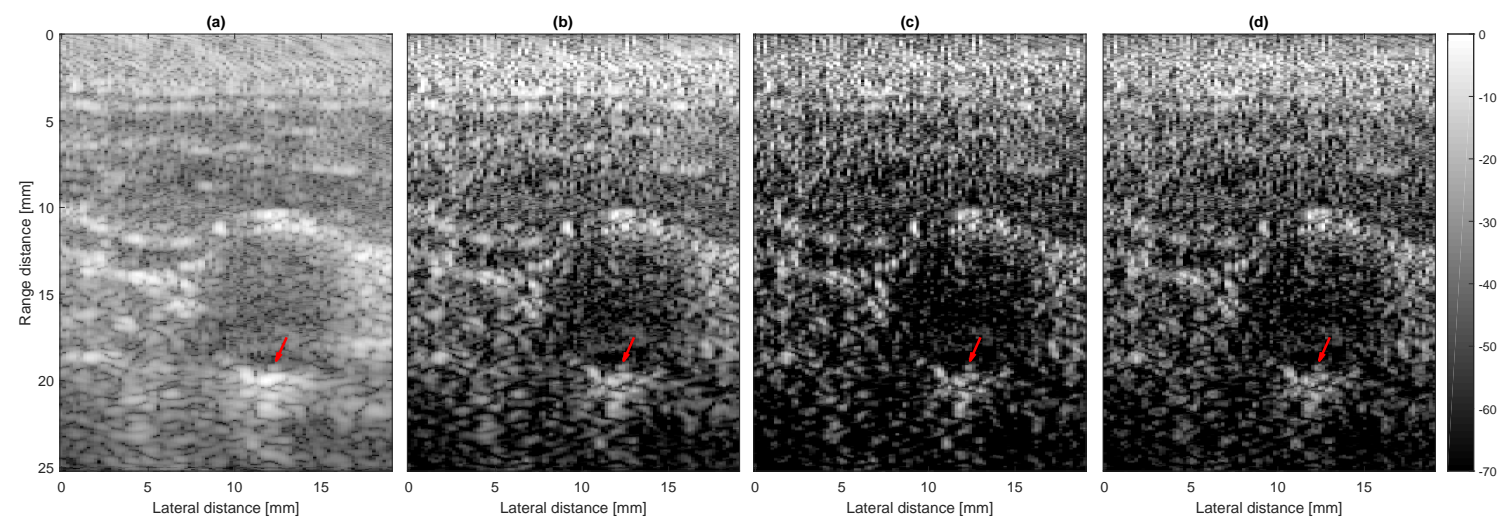

Figure 8. B-mode images of carotid artery obtained (a) with DAS; (b) with mAPES beamforming without gPCF, and those with gPCFs estimated (c) before and (d) after applying the beamformer weights.

\section{Discussion}

In the present study, we tried to combine the modified adaptive beamformer with the phase coherence weighting. From the experiments using a wire phantom, the lateral full width at half maxima obtained by the proposed method was better than conventional DAS beamforming and adaptive beamforming only. Also, in a B-mode image shown in Figure 2c, the speckles in diffuse scattering medium in the axial deep region were more clearly resolved than in Figure 2b. Furthermore, as shown 
in Figures $2 \mathrm{c}$ and $6 \mathrm{a}$, obtained results show that the visibility of echoes from diffuse scattering medium was significantly improved by applying the gPCF to the outputs of the sub-aperture beamformers.

In the present study, we also tried to estimate the PCF using the phase variance of the outputs of the sub-aperture beamformers, which were subjected to the weight vector determined by the mAPES beamformer. In such a case, as shown in the B-mode image in Figure $2 \mathrm{~d}$, the spatial resolution became worse as compared to that shown in Figure 2c, in which the PCF was estimated from the outputs of the sub-aperture beamformers before applying the adaptive weights. The mAPES beamformer changes the amplitude and phase of the outputs of the sub-aperture beamformers adaptively so that the lateral resolution is optimized. In Figure $4 a, b$, obtained results show that the phase rotation was induced by the adaptive beamformer weights even with the echo signals from a strong scatterer and, therefore, the gPCF became low. With respect to the point target at an axial depth of $12 \mathrm{~mm}$, a point scatterer depicted in Figure 2d was blurred as compared to that depicted in Figure 2c. This is because the peak value of the echo from the point scatterer decreased by the gPCF from echo signals after adaptive weighting but the phase variance of the signals other than the peak position is originally large and adaptive weights do not change the gPCF value significantly.

From the experimental results on a cyst phantom, which are shown in Figure 5a-d, image contrast was improved using the gPCF estimated before and after applying the adaptive beamformer weights. Both the adaptive beamformer and the PCF were proposed for improvement of the spatial resolution but improvement of contrast was accomplished by applying the gPCF to the output of the modified adaptive beamformer. From the experimental results on two phantoms, the gPCF evaluated using echo signals subjected to mAPES beamformer weights was shown to give worse results than that evaluated using echo signals without mAPES beamformer weights. Consequently, evaluating the gPCF using echo signals subjected to adaptive beamformer weights is not preferred.

In the B-mode images in Figure 2, a point target at a depth of about $7 \mathrm{~mm}$ is somewhat blurred also in the B-mode image obtained by DAS beamforming (Figure 2a). In the near field, the ultrasound field fluctuates significantly due to interference among ultrasonic waves emitted from the transducer [38], and the performance of image formation is degraded. The performance of the proposed method could be degraded because such interference would also affect the proposed method, which uses the amplitude and phase information of the measured ultrasonic signal. Therefore, we need more improvements in the beamformation process for near-field imaging.

In the proposed method, the penetration depths in the obtained B-mode images were about $30 \mathrm{~mm}$ because the center frequency of the linear array probe used in the present study was $7.5 \mathrm{MHz}$ and it was fabricated for imaging of superficial tissues. To increase the penetration depth up to $10-20 \mathrm{~cm}$, we need to use an ultrasonic probe at a lower center frequency. The performance of the proposed method on such probes at lower center frequencies will be investigated in our future work.

In the present study, the B-mode images obtained without the sub-aperture beamforming were also evaluated as the previously proposed method. With respect to the lateral resolution, the appearance of the fine wire depicted in Figure 6a does not seem to change as compared to Figure 2c. On the other hand, the speckle visibility in the obtained B-mode image was degraded. In ultrafast ultrasound imaging, the adaptive beamformer incorporating with the coherence factor degrades the speckle visibility in the obtained image [39]. In the present study, the output signals from the sub-aperture beamformers were used in estimation of both the beamformer weights and the gPCF, and experimental results obtained with sub-aperture beamforming show that such an implementation is effective to avoid degradation of the speckle visibility while maintaining the improvement of the spatial resolution. With respect to image contrast, better contrast was obtained by the proposed method without sub-aperture beamforming. However, when the proposed method is performed without sub-aperture beamforming, the dimension of the covariance matrix becomes large and high computational cost is required to calculate the inverse matrix of the covariance matrix. The sub-aperture beamforming can reduce the dimension of the covariance matrix, and accordingly, the computation time becomes shorter [27]. 
As described above, although the proposed method needs to carry out an additional processing for weighting the coherence factor on the conventional modified adaptive beamforming, the gPCF estimated with sub-aperture beamforming is a computationally effective method. In the present study, better lateral resolution and contrast were realized with slightly increased the processing time compared to the mAPES beamformer only. The computation time of the proposed method was $339 \%$ of the conventional DAS beamformer, when an Intel(R) Core(TM) i5-6500 CPU was used with 8 GB of RAM. The proposed method has potential to be used in practical applications by incorporating parallel processing techniques with the proposed method.

\section{Conclusions}

The spatial resolution and image contrast are important factors determining the quality of an ultrasound B-mode image. In the present study, we introduced the modified adaptive beamformer enhanced by PCF for further improvement of image quality. From the results of the phantom experiments, the spatial resolution evaluated by the lateral full widths at half maxima of echoes from point targets and image contrast were improved by the proposed method.

Acknowledgments: This study was supported by JSPS KAKENHI Grant Numbers JP17H03276 and JP15K13995.

Author Contributions: Hideyuki Hasegawa conceived and designed the experiments. Michiya Mozumi analyzed the data. Michiya Mozumi and Hideyuki Hasegawa wrote the paper.

Conflicts of Interest: The authors declare no conflict of interest.

\section{References}

1. Shattuck, D.P.; Weinshenker, M.D. Explososcan: A parallel processing technique for high speed ultrasound imaging with linear phase array. J. Acoust. Soc. Am. 1984, 75, 1273-1282.

2. Tanter, M.; Fink, M. Ultrafast imaging in biomedical ultrasound. IEEE Trans. Ultrason. Ferroelectr. Freq. Control 2014, 61, 102-119.

3. Tanter, M.; Bercoff, J.; Sandrin, L.; Fink, M. Ultrafast compounding imaging for 2-D motion vector estimation: application to transient elastography. IEEE Trans. Ultrason. Ferroelectr. Freq. Control 2002, 49, 1363-1374.

4. Bercoff, J.; Tanter, M.; Fink, M. Supersonic shear imaging: A new technique for soft tissue elasticity mapping. IEEE Trans. Ultrason. Ferroelectr. Freq. Control 2004, 51, 396-409.

5. Honjo, Y.; Hasegawa, H.; Kanai, H. Accurate ultrasonic measurement of myocardial regional strain rate at high temporal and spatial resolutions. In Proceedings of the 2008 IEEE International Ultrasonics Symposium (IUS), Beijing, China, 2-5 November 2008; pp. 1995-1998.

6. Provost, J.; Nguyen, V.T.-H.; Legrand, D.; Okrasinski, S.; Costet, A.; Gambhir, A.; Garan, H.; Konofagou, E.E. Electromechanical wave imaging for arrhythmias. Phys. Med. Biol. 2011, 56, L1-L11.

7. Cikes, M.; Tong, L.; Sutherland, G.R.; D’hooge, J. Ultrafast cardiac ultrasound imaging: Technical principles, applications, and clinical benefits. JACC Cardiovasc. Imaging 2014, 7, 812-823.

8. Udesen, J.; Gran, F.; Hansen, K.L.; Jensen, J.A.; Thomsen, C.; Nielsen M.B. High frame-rate blood vector velocity imaging using plane waves: Simulations and preliminary experiments. IEEE Trans. Ultrason. Ferroelectr. Freq. Control 2008, 55, 1729-1743.

9. Hasegawa, H.; Kanai, H. Simultaneous imaging of artery wall strain and blood flow by high frame rate acquisition of RF signals. IEEE Trans. Ultrason. Ferroelectr. Freq. Control 2008, 55, 2626-2639.

10. Bercoff, J.; Montaldo, G.; Loupas, T.; Savery, D.; Mézière, F.; Fink, M.; Tanter, M. Ultrafast compound Doppler imaging: providing full blood flow characterization. IEEE Trans. Ultrason. Ferroelectr. Freq. Control 2011, 58, 134-147.

11. Yiu, B.Y.; Yu, A.C. High-frame-rate ultrasound colorencoded speckle imaging of complex flow dynamics. Ultrasound Med. Biol. 2013, 39, 1015-1025.

12. Takahashi, H.; Hasegawa, H.; Kanai, H. Temporal averaging of two-dimensional correlation functions for velocity vector imaging of cardiac blood flow. J. Med. Ultrason. 2015, 42, 323-330.

13. Takahashi, H.; Hasegawa, H.; Kanai, H. Echo motion imaging with adaptive clutter filter for assessment of cardiac blood flow. Jpn. J. Appl. Phys. 2015, 54, doi:10.7567/JJAP.54.07HF09. 
14. Jensen, J.A.; Nikolov, S.I.; Yu, A.C.H.; Garcia, D. Ultrasound vector flow imaging-Part II: Parallel system. IEEE Trans. Ultrason. Ferroelectr. Freq. Control 2016, 63, 17221732.

15. Montaldo, G.; Tanter, M.; Bercoff, J.; Benech, N. Fink, M. Coherent plane-wave compounding for very high frame rate ultrasonography and transient elastography. IEEE Trans. Ultrason. Ferroelectr. Freq. Control 2009, $56,489-506$.

16. Denarie, B.; Tangen, T.A.; Ekroll, I.K.; Rolim, N.; Torp, H.; Bjåstad, T.; Lovstakken, L. Coherent plane-wave compounding for very high frame rate ultrasonography of rapidly moving targets. IEEE Trans. Med. Imaging 2013, 32, 1265-1276.

17. Jensen, J.A.; Nikolov, S.I.; Gammelmark, K.L.; Pedersen, M.H. Synthetic aperture ultrasound imaging. Ultrasonics 2006, 44, e5-e15.

18. Hasegawa, H.; De Korte, C.L. Impact of element pitch on synthetic aperture ultrasound imaging. J. Med. Ultrason. 2016, 43, 317-325.

19. Veen, B.D.V.; Buckley, K.M. Beamforming: A versatile approach to spatial filtering. IEEE ASSP Mag. 1988, 5, 4-24.

20. Capon, J. High-resolution frequency-wavenumber spectrum analysis. Proc. IEEE 1969, 57, 1408-1418.

21. Sasso, M.; Cohen-Bacrie, C. Medical ultrasound imaging using the fully adaptive beamformer. In Proceedings of the IEEE International Conference on Acoustics, Speech, and Signal Processing, Philadelphia, PA, USA, 23 March 2005; pp. 489-492.

22. Synnevåg, J.F.; Austeng, A.; Holm, S. Adaptive beamforming applied to medical ultrasound imaging. IEEE Trans. Ultrason. Ferroelectr. Freq. Control 2007, 54, 1606-1613.

23. Holfort, I.K.; Gran, F.; Jensen, J.A. Broadband minimum variance beamforming for ultrasound imaging. IEEE Trans. Ultrason. Ferroelectr. Freq. Control 2009, 56, 314-325.

24. Synnevåg, J.F.; Austeng, A.; Holm, S. Benefits of minimum-variance beamforming in medical ultrasound imaging. IEEE Trans. Ultrason. Ferroelectr. Freq. Control 2009, 56, 1868-1879.

25. Blomberg, A.E.A.; Holfort, I.K.; Austeng, A.; Synnevåg, J.F.; Jensen, J.A. APES beamforming applied to the ultrasound imaging. In Proceedings of the 2009 IEEE International Ultrasonics Symposium (IUS), Rome, Italy, 20-23 September 2009; pp. 2347-2350.

26. Hasegawa, H.; Kanai, H. Effect of element directivity on adaptive beamforming applied to high-frame-rate ultrasound. IEEE Trans. Ultrason. Ferroelectr. Freq. Control 2015, 62, 511-523.

27. Hasegawa, H. Improvement of penetration of modified amplitude and phase estimation beamformer. J. Med. Ultrason. 2017, 44, 3-11.

28. Hasegawa, H. Apodized adaptive beamformer. J. Med. Ultrason. 2017, 44, 155-165.

29. Hasegawa, H. Adaptive beamforming applied to transverse oscillation. In Proceedings of the 2017 IEEE International Ultrasonics Symposium (IUS), Washington, DC, USA, 6-9 September 2017; pp. 1-4.

30. Li, P.-C.; Li, M.-L. Adaptive imaging using the generalized coherence factor. IEEE Trans. Ultrason. Ferroelectr. Freq. Control 2003, 50, 128-141.

31. Camacho, J.; Parrilla, M.; Fritsch, C. Phase coherence imaging. IEEE Trans. Ultrason. Ferroelectr. Freq. Control 2009, 56, 958-974.

32. Asl, B.M.; Mahloojifar, A. Minimum variance beamforming combined with adaptive coherence weighting applied to medical ultrasound imaging. IEEE Trans. Ultrasonics. Ferroelectr. Freq. Control 2009, 56, 1923-1931.

33. Nilsen, C.-I.C.; Holm, S. Wiener beamforming and the coherence factor in ultrasound imaging. IEEE Trans. Ultrasonics. Ferroelectr. Freq. Control 2010, 57, 1329-1346.

34. Chen, H.; Li, M. Improved high axial resolution ultrasound imaging using spectral whitening and minimum-variance based coherence weighting. In Proceedings of the 2017 IEEE International Ultrasonics Symposium (IUS), Washington, DC, USA, 6-9 September 2017; pp. 1-4.

35. Hasegawa, H.; Kanai, H. Effect of subaperture beamforming on phase coherence imaging. IEEE Trans. Ultrason. Ferroelectr. Freq. Control 2014, 61, 1779-1790.

36. Hasegawa, $\mathrm{H}$. Enhancing effect of phase coherence factor for improvement of spatial resolution in ultrasonic imaging. J. Med. Ultrason. 2016, 43, 19-27.

37. Fujita H.; Hasegawa, H. Effect of frequency characteristic of excitation pulse on lateral spatial resolution in coded ultrasound imaging. Jpn. J. Appl. Phys. 2017, 56, doi:10.7567/JJAP.56.07JF16. 
38. Franco, E.E.; Andrade, M.A.B.; Adamowski, J.C.; Buiochi, F. Acoustic beam modeling of ultrasonic transducers and arrays using the impulse response and the discrete representation methods. J. Braz. Soc. Mech. Sci. Eng. 2011, 33, 408-416.

39. Varray F.; Kalkhoran M.A.; Vray D. Adaptive minimum variance coupled with sign and phase coherence factor in IQ domain for plane wave beamforming. In Proceedings of the 2016 IEEE International Ultrasonics Symposium (IUS), Tours, France, 18-21 September 2016; pp. 1-4.

(C) 2018 by the authors. Licensee MDPI, Basel, Switzerland. This article is an open access article distributed under the terms and conditions of the Creative Commons Attribution (CC BY) license (http:/ / creativecommons.org/licenses/by/4.0/). 